\title{
Editorial Introduction to the Special Issue: Terahertz Metamaterials and Photonic Crystals
}

\author{
Ibraheem Al-Naib ${ }^{1}$. \\ Withawat Withayachumnankul ${ }^{2}$
}

Received: 10 July 2017 / Accepted: 11 July 2017 /

Published online: 26 July 2017

(C) Springer Science+Business Media, LLC 2017

The dawn of this century saw an emerging field of engineered materials called metamaterials. The concept promises a great degree of freedom for wave manipulation with these artificial materials across the electromagnetic spectrum. In the last decade, there has been a rapid progress in this field towards many applications. Underlying those applications are metamaterial-based components such as filters, absorbers, antenna structures, sensors, and high-frequency modulators. For terahertz (THz) science and technology, these metamaterials have been at the core, as various functionalities cannot be derived from natural materials with relatively weak electromagnetic responses. Motivated by this rapid development, this special issue features some latest progresses in terahertz metamaterials and photonic crystals. It consists of ten invited articles, the content of which is briefly outlined below. These contributions represent some of the ongoing research in this area.

1. Tailoring terahertz propagation by phase and amplitude control in metasurfaces by J. Zheng, X. Zhang, L. Liu, Q. Li, L. Singh, J. Han, F. Yan, and W. Zhang (a collaboration between USA and Chinese universities) presents a review of their recent progress in metasurface-mediated propagation control of terahertz free-space and surface waves.

2. Review on polarization selective terahertz metamaterials: from chiral metamaterials to stereometamaterials by E. Philip, M. Z. Güngördü, S. Pal, P. Kung,

Ibraheem Al-Naib

iaalnaib@uod.edu.sa

Withawat Withayachumnankul

withawat@adelaide.edu.au

1 Biomedical Engineering Department, College of Engineering, University of Dammam,

Dammam 31441, Kingdom of Saudi Arabia

2 School of Electrical and Electronic Engineering, The University of Adelaide, Adelaide,

SA 5005, Australia 
and Margaret Kim (the University of Alabama, USA) thoroughly reviewed recent development of sophisticated metamaterial structures called chiral metamaterials and stereometamaterials in the terahertz region and their abilities to perform as polarization waveplates, filters, and modulators.

3. Recent progress in terahertz metasurfaces by I. Al-Naib and W. Withayachumnankul (University of Dammam, Saudi Arabia, and The University of Adelaide, Australia) reviews recent progress in terahertz metamaterials relating to wavefront control, high-Q sensing, and graphene-based structures.

4. Terahertz sensor using photonic crystal cavity and resonant tunneling diodes by K. Okamoto, K. Tsuruda, S. Diebold, S. Hisatake, M. Fujita, and T. Nagatsuma (Osaka University and ROHM Co. Ltd., Japan) demonstrates a photonic crystal cavity integrated with resonant tunneling diodes for a source and detector. The integrated system achieves a quality factor of more than 10,000 for sensing applications.

5. Influence of distance between metal squares in checkerboard patterns on transmittance characteristics in the infrared region by T. Higashira, T. Kageyama, K. Kashiwagi, H. Miyashita, K. Takano, M. Nakajima, and S.-S. Lee (Tottori University and Osaka University, Japan) investigates the influence of the metal square configuration in self-complementary metallic checkerboard patterns in the infrared region.

6. Direct measurements of terahertz meta-atoms with near-field emission of terahertz waves by K. Serita, J. Darmo, I. Kawayama, H. Murakami, and M. Tonouchi (Osaka University, Japan, and Vienna University of Technology, Austria) presents direct measurements of terahertz meta-atoms by using locally generated terahertz waves in the near-field region.

7. Narrowband metamaterial absorber for terahertz secure labeling by M. Nasr, J. T. Richard, S. A. Skirlo, M. S. Heimbeck, J. D. Joannopoulos, M. Soljacic, H. O. Everitt, and L. Domash (Triton Systems Inc.; Massachusetts Institute of Technology, U.S. Army Aviation and Missile RD\&E Center, and IERUS Technologies, USA) describes flexible metamaterial films for securely labeling objects.

8. Metamaterial demonstrates both a high refractive index and extremely low reflection in the $0.3-\mathrm{THz}$ band by $\mathrm{K}$. Ishihara and T. Suzuki (Ibaraki University and Tokyo University of Agriculture and Technology, Japan) reports the implementation of an unprecedented metamaterial with a high effective refractive index of $6.66+j 0.123$, an extremely low reflection power of $1.16 \%$, and a high figure of merit (FOM $=\left|n_{\text {real }} / n_{\text {imag }}\right|$ ) of above 300 at a frequency of $0.3 \mathrm{THz}$.

9. A brief review on metamaterial-based vacuum electronics for terahertz and microwave science and technology by T. Matsui (Mie University, Japan) summarizes recent research activities on metamaterial-inspired vacuum electronics for microwave and $\mathrm{THz}$ science and technology.

10. Fiber drawn metamaterial for $\mathrm{THz}$ waveguiding and imaging written by $\mathrm{S}$. Atakaramians, A. Stefani, H. Li, Md. S. Habib, J. G. Hayashi, A. Tuniz, X. Tang, J. Anthony, R. Lwin, A. Argyros, S. C. Fleming, and B. T. Kuhlmey (The University of Sydney, Australia; Technical University of Denmark, Denmark; and Beijing Jiaotong University, China) reviews their research activities in 
metamaterials fabricated by fiber drawing for terahertz waveguides and imaging applications.

The guest editors would like to thank all contributors and reviewers for their valuable contributions. We hope that the readers will find this special issue useful for their research. 